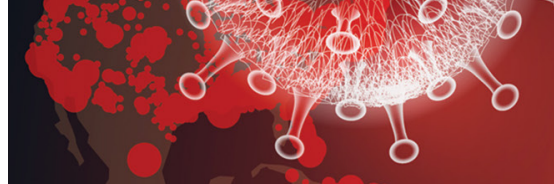

\title{
Is it safe to be pregnant during the COVID-19 pandemic?
}

\author{
Expert answers to 8 of your most pressing questions about \\ coronavirus disease 2019 and pregnancy, including vaccination
}

\author{
Malavika Prabhu, MD
}

IN THIS ARTICLE

Newborn

outcomes

page 29

\section{Outpatient management page 30}

\section{Vaccine}

safety

page 31

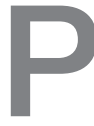

regnant women, or women considering pregnancy, want to know-is pregnancy safe in the midst of the coronavirus disease 2019 (COVID-19) pandemic? In this article, I tackle common questions facing reproductive-aged or pregnant women and their providers.

\section{What are the risks of COVID-19 in pregnancy?}

A large, national prospective cohort study of outpatient pregnant and recently postpartum women with the diagnosis of suspected or confirmed COVID-19 demonstrated that many affected women have mild illnesses, with typical symptoms including cough, sore throat, body aches, fever, and headache. ${ }^{1}$ Although symptoms were most common within the first 3 weeks of presentation, approximately $25 \%$ of women had a protracted course of symptoms (8 or more weeks). As this cohort disproportionately enrolled outpatients, it is important to note that many women had mild illnesses, which

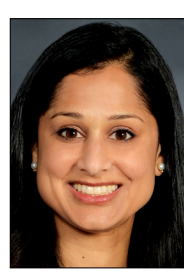

Dr. Prabhu is from the Division of Maternal-Fetal Medicine, Department of Obstetrics \& Gynecology, Weill Cornell Medicine,

New York City, New York.

The author reports no financial relationships relevant to this article.

doi: 10.12788 /obgm.0063 is the most likely course of infection in otherwise healthy, young women.

Data on the impact of COVID-19 on rates of miscarriage and birth defects are limited, yet the published reports are reassuring, with no increased risks of miscarriage, and no clear signal for birth defects. ${ }^{2}$

In a prospective cohort study across 3 New York City institutions when universal severe acute respiratory syndrome coronavirus 2 (SARS-CoV-2) testing was recommended upon admission for delivery, approximately $80 \%$ of women who were positive were asymptomatic. ${ }^{3}$ Maternal outcomes generally were reassuring, with no patients experiencing severe or critical illness. There were no differences in preterm delivery rates by SARS-CoV-2 status, but the rate of cesarean delivery was higher among women with COVID-19, for unclear reasons. Most notably, the rate of postpartum complications was $13 \%$ among women with COVID-19, versus $2.5 \%$ among women without COVID-19. These complications included readmission for worsening COVID-19, postpartum hypoxia, and postpartum fever.

A recent prospective cohort study from 1 institution in Texas similarly demonstrated favorable maternal outcomes with COVID19 , with $95 \%$ of women with asymptomatic or mild illness, and no differences in adverse pregnancy outcomes between COVID-19positive and COVID-19-negative women, including cesarean delivery rate. ${ }^{4}$

Finally, certain characteristics increase the risk of COVID-19 among pregnant 


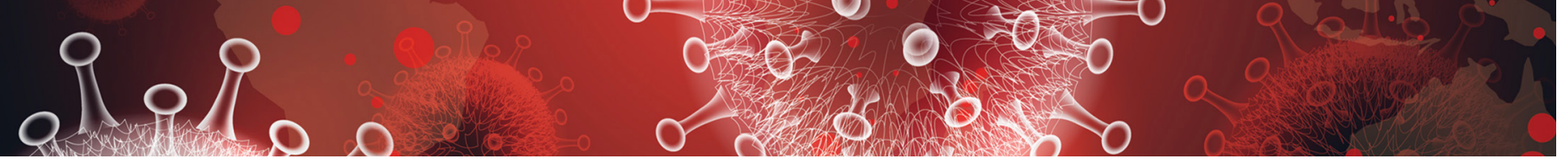

women and nonpregnant individuals alike. In a nationwide prospective cohort from the United Kingdom, medical comorbidities including obesity, diabetes (gestational or pregestational), hypertension, as well as Black or other minority ethnicities are associated with COVID-19. ${ }^{5}$ This is particularly notable given universal health insurance in the United Kingdom. Other data have also confirmed that women with comorbidities, women of Black or Hispanic ethnicity, and women with lower socioeconomic status, are at increased risk of COVID-19. ${ }^{3,6,7}$

\section{Is COVID-19 worse in pregnancy?}

Given the well-documented risks of COVID19 outside of pregnancy, is COVID-19 worse in a pregnant woman than in a nonpregnant woman? The most recent guidance from the Centers for Disease Control and Prevention (CDC) from November 2020 suggests that pregnant women are at increased risk for severe illness. ${ }^{8}$ However, it is important to understand the design of this study in order to appreciate its implications. Laboratory confirmed SARS-CoV-2 in the United States is systematically reported to the CDC. Among women aged 15-44 years with such confirmation, data on pregnancy status were available for $35.5 \%$, almost $90 \%$ of whom were symptomatic. Within this cohort of largely symptomatic pregnant women, risks of intensive care unit (ICU) admission, invasive ventilation, and use of extracorporeal membrane oxygenation (ECMO) were approximately 2 to 3 times higher for pregnant women than for nonpregnant women. The absolute risks, however, were low. The risk of ICU admission for symptomatic pregnant women was approximately $1 \%$; the risk of invasive ventilation, $0.3 \%$; and the risk of ECMO, $0.1 \%$.

Moreover, the lack of uniform data capture on pregnancy status for all women ages 15-44 years may skew the population with known pregnancy status to be sicker and, thus, may bias the results toward increased risks. Nevertheless, there is consistency in several publications with different data

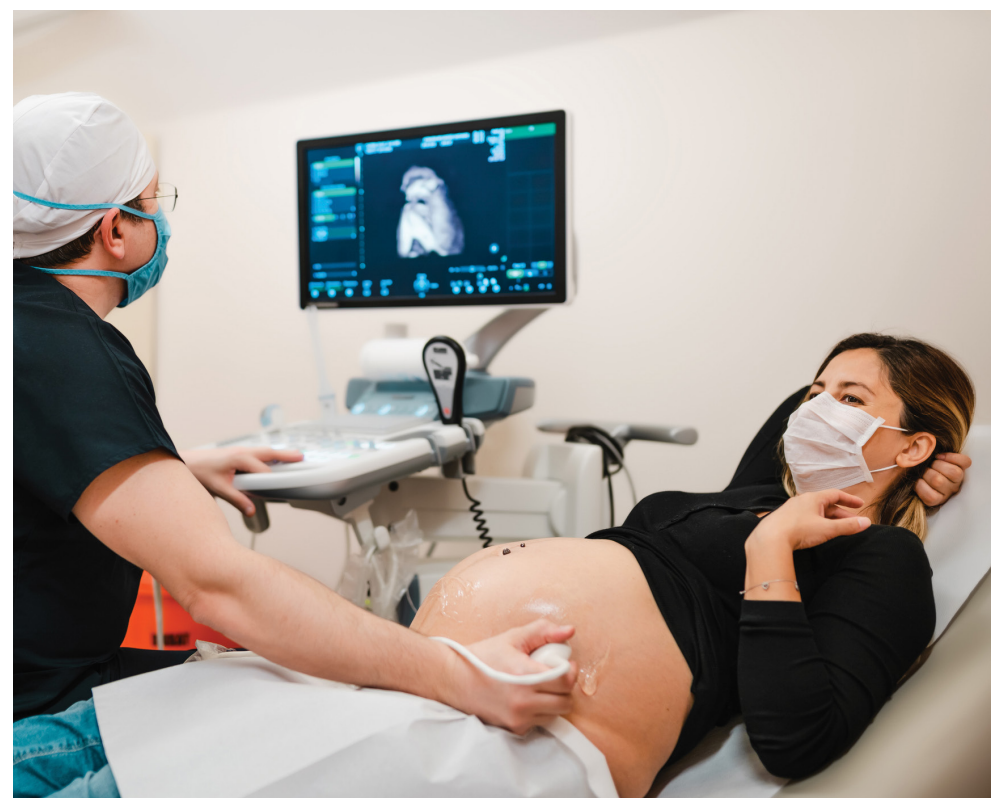

sources, all of which suggest pregnancy is an independent risk factor for increased severity of COVID-19.9-11 Additionally, women with medical comorbidities (such as pregestational or gestational diabetes or obesity) are more likely to have severe COVID- 19 .

\section{What are newborn outcomes if COVID-19 is diagnosed during pregnancy?}

Two large cohorts of newborns, disproportionately term infants, from the first wave of the pandemic in New York City, have reassuring news. In one cohort of 101 infants born at 2 New York City institutions to SARS-CoV-2positive mothers, 2 neonates were diagnosed with SARS-CoV-2 during the immediate postnatal period. ${ }^{12}$ Neither infant demonstrated clinical COVID-19. In another cohort of 120 infants born at 3 other New York City institutions to SARS-CoV-2-positive mothers and tested systematically within 24 hours of life, 5-7 days of life, and 14 days of life, there were no neonates who tested positive for SARS-CoV-2 at the initial time point. Among the 79 infants who had testing at 5-7 days of life and the 72 tested at 14 days of life, there were no infants positive for SARS-CoV-2. ${ }^{13}$ It is important to note that case reports and
FAST

TRACK

Overwhelming evidence suggests that the risk of vertical transmission of SARS-CoV-2 is very low 
FAST

TRACK

As postpartum
VTE is a risk
for women
with COVID-19,
prophylactic
anticoagulation
is recommended
for 2 weeks
after vaginal
and 6 weeks
after cesarean
delivery

As postpartum

VTE is a risk

for women

with COVID-19,

prophylactic

anticoagulation

is recommended

for 2 weeks

after vaginal

and 6 weeks

delivery small case series have demonstrated some convincing evidence of vertical transmission. However, the overwhelming evidence suggests this risk is very low.

\section{What is a reasonable outpatient setting-approach to managing COVID-19 in a pregnant woman?}

Women should be counseled to quarantine for 10 to 14 days from symptom onset or, if asymptomatic, from positive polymerase chain reaction (PCR) test. Warning signs of worsening COVID-19 disease should be reviewed. Serial telemedicine follow-up for 10 to 14 days is recommended to ensure clinical stability and continued management as an outpatient. A home pulse oximeter is also recommended. Women should be advised to check their oxygen saturation daily and to call if oxygen saturation becomes less than $93 \%$. Supportive care is recommended.

If delay in obstetric care may result in adverse pregnancy outcomes (for instance, postponing indicated fetal surveillance), obstetric care should be delivered, with appropriate personal protective equipment for health care workers and minimization of exposure of other pregnant women to the infected patient. Appointments should be scheduled at the end of the day.

During influenza season, women should receive empiric oseltamivir treatment (75 mg twice a day) per CDC guidelines for symptoms that may also be consistent with influenza, regardless of testing.

Prophylactic anticoagulation is not indicated for pregnant antepartum women who do not require inpatient care.

If inpatient care is required, management is individualized.

The approach to prenatal care after resolution of COVID-19 is not evidence-based. At my institution, all patients have a detailed mid-trimester anatomic evaluation, but if this is not routine, a detailed anatomic ultrasound (Current Procedural Terminology code 76811) may be considered. Additionally, for women with COVID-19 we perform one third-trimester growth ultrasound to screen for fetal growth restriction, on the basis of several placental studies demonstrating clots on the fetal or maternal side of the placenta. ${ }^{3,14}$ Routine antenatal testing in the absence of growth restriction, or other comorbid conditions for which testing occurs, is not recommended.

\section{What if asymptomatic or mild COVID-19 is diagnosed at the time of delivery? What is reasonable management?}

Asymptomatic or mildly symptomatic COVID-19 should not alter obstetric management, beyond appropriate use of personal protective equipment. Delayed cord clamping is also reasonable, if there are no other contraindications, as there is no documented harm associated with this practice among women with COVID-19.

Women with COVID-19 may be at higher risk for venous thromboembolic events in the postpartum period. At my institution, prophylactic postpartum anticoagulation is recommended for 2 weeks after vaginal delivery, and 6 weeks after cesarean delivery.

During the postpartum hospitalization, given reassuring data about vertical transmission and postnatal horizontal transmission risks, babies may room in with mothers in a single private room, if rooming-in is the current standard of care-as long as the mother and newborn do not require higher levels of care. Mothers should wear a mask and use hand hygiene when in contact with the baby. Skin-to-skin and breastfeeding or infant feeding of breast milk are appropriate practices to continue. There is no evidence to suggest that transmission of COVID-19 can occur via breastmilk; however, given the close contact inherent in breastfeeding, transmission through direct contact or maternal respiratory droplets is possible, and thus maternal use of masks and hand hygiene is recommended. When not feeding, the infant should be 6 feet away, and if possible, in an isolette. 


\section{When can individuals with COVID-19 discontinue transmission precautions or "home quarantine"?}

For women with mildly symptomatic COVID-19 and without immunocompromise, home quarantine can be discontinued 10 days after onset of symptoms as long as there has been symptom improvement and no fever for at least 24 hours without the use of antipyretics. For immunocompetent women with incidentally diagnosed asymptomatic COVID-19, home quarantine can be discontinued 10 days after the positive test was obtained. Pregnancy in and of itself is not an immunocompromising condition..$^{15,16}$

For women with severe or critical COVID-19, who were hospitalized due to their clinical status, home quarantine can be discontinued when at least 10 days, and up to 20 days, after onset of symptoms and with symptom improvement and with no fever for at least 24 hours, without the use of antipyretics. Local hospital infection control experts may be able to guide the recommended practice for your site better, based on local information. ${ }^{15,16}$

Repeating a PCR test to discontinue home quarantine is not recommended in most circumstances, as individuals may have prolonged shedding of noninfectious particles in their nasopharynx. Immunocompromise may be one exception to this general guidance, but consultation with local hospital infection control experts will help guide management. ${ }^{15,16}$

\section{Should women get pregnant during the COVID-19 pandemic?}

Every pandemic has its own set of implications for the health of the mother, fetus, or both, and COVID-19 is no exception. While there are risks, described above, to mother and fetus, these risks are not so catastrophic as to strongly and directively recommend a patient not become pregnant. ${ }^{17}$ Moreover, the last several months of the pandemic have demonstrated that consistent mask usage, social distancing, and hand hygiene, are effective methods of preventing the acquisition of COVID-19. All of these risk-reducing strategies are available to pregnant women. Finally, accessing care during a pandemic in a hospital setting does not also pose a risk for acquisition of SARS-CoV-2. ${ }^{18}$

\section{Is the COVID-19 vaccine safe for pregnant or postpartum/ lactating women?}

On December 11, 2020, the US Food and Drug Administration (FDA) issued emergency use authorization (EUA) for the Pfizer-BioNtech mRNA vaccine (BNT 162b2) against COVID-19, for individuals aged 16 and older as a 2-dose series given 21 days apart. Among the more than 40,000 individuals in the trial that led to this EUA, vaccine efficacy was $95 \% .^{19}$ Adverse effects included fatigue and headache most commonly, with $16 \%$ of vaccine recipients experiencing fever after the second dose. Follow-up regarding safety is planned for 2 years by the manufacturer, in addition to safety monitoring by pre-existing national systems.

On December 18, 2020, the FDA announced EUA for Moderna's mRNAbased vaccine, mRNA-1273, in men and women aged 18 and older. This is a 2-dose series given 28 days apart. The vaccine efficacy has been reported at $94.5 \%$, with the most common adverse effects being injection site pain, tiredness, headache, muscle pain, chills, joint pain, swollen lymph nodes in the same arm as the injection, nausea and vomiting, and fever. ${ }^{20,21}$ The phase 3 trial is ongoing.

Despite the speed with which these effective vaccines were developed, it is important to note that all regulatory and safety steps mandated for the development of any vaccine were met for these two, as well as for other COVID-19 vaccinations that will similarly receive EUA from the FDA.

In the EUA for BNT 162b2, the specific language regarding pregnant and lactating women recommends that patients and

\section{FAST} TRACK

Two mRNA vaccines are currently authorized for EUA use, with efficacy rates reported as $95 \%$ and $94.5 \%$ with each 2-dose series 
FAST

TRACK

ACOG recom-
mends that

COVID-19 vaccines not be withheld

from pregnant or lactating women.

Trials specific to

pregnant women

will begin in early

2021 and will offer more information

in this patient

group. providers have an individualized conversation about vaccination. In the data presented to the FDA for the Pfizer-BioNtech mRNA vaccine, a limited number of pregnant women received either the vaccine (12 women) or placebo (11 women), with no long-term follow-up data available to characterize either maternal or fetal benefits and risks. The mechanism of action of an mRNA vaccine is to induce the cytoplasmic machinery within cells to create the coronavirus spike protein, which then allows the body's immune system to create antibodies against this protein and confer protection accordingly. While the above mechanism is not theorized to result in different outcomes or different efficacy, the safety for the pregnant woman and fetus are unknown. It is not believed that vaccination during lactation would cause any adverse outcomes to a neonate, and lactating women do not need to interrupt or discontinue breast milk production in order to receive the vaccine.

The American College of Obstetricians and Gynecologists (ACOG) released a Practice Advisory on December 13, 2020, regarding their recommendations. ${ }^{22}$ ACOG recommends that vaccines against COVID19 not be withheld from pregnant or lactating women, if they might otherwise meet criteria for and have access to vaccination. Currently, the CDC's Advisory Committee on Immunization Practices (ACIP) stated that health care workers and long-term care facility residents represent priority groups to vaccinate in the initial phases of vaccination, given limitations in supply. ${ }^{23}$ This recommendation is likely to be updated frequently as additional vaccines become available. Shared decision-making between patient and provider may help the patient to make the best decision for herself, but provider input is not required prior to a pregnant woman being vaccinated.

Additional animal data evaluating adverse effects on the reproductive system from developmental and reproductive toxicity (DART) studies for both mRNA vaccines should be available in the coming weeks, which may aid in the counseling of reproductive-aged women.

Vaccine trials to specifically enroll pregnant women are set to begin in early 2021, and more data will certainly inform the conversation between patient and provider regarding risks and benefits.

\section{Conclusions}

While the absolute risks of COVID-19 to mothers, fetuses, and neonates is low, pregnancy is a risk factor for severe disease. Many pregnant women with COVID19 can be safely followed as outpatients via telemedicine, and supportive care is recommended. Inpatient care should be individualized. Pregnancy during the COVID-19 pandemic should be not be absolutely discouraged; instead, a conversation about risk mitigation should be undertaken. The COVID-19 vaccine is available to pregnant and lactating women, and the decision to choose vaccination in pregnancy is in the purview of the patient, in consultation with her physician.
References

1. Afshar Y, Gaw SL, Flaherman VJ, et al. Clinical presentation of coronavirus disease 2019 (COVID-19) in pregnant and recently pregnant people. Obstet Gynecol. 2020;128:1117-1125.

2. Cosma S, Carosso AR, Cusato J, et al. Coronavirus disease 2019 and first-trimester spontaneous abortion: a casecontrol study of 225 pregnant patients. Am J Obstet Gynecol. 2020;S0002-9378:31177-7. doi: 10.1016/j.ajog.2020.10.005.

3. Prabhu M, Cagino K, Matthews KC, et al. Pregnancy and postpartum outcomes in a universally tested population for SARS-CoV-2 in New York City: a prospective cohort study. BJOG. 2020;127:1548-1556.

4. Adhikari E, Moreno W, Zofkie AC, et al. Pregnancy outcomes among women with and without severe acute respiratory syndrome coronavirus 2 infection. JAMA Netw Open. 2020;3:e2029256.
5. Knight M, Bunch K, Vousden B, et al; UK Obstetric Suveillance System SARS-CoV-2 Infection in Pregnancy Collaborative Group. Characteristics and outcomes of pregnant women admitted to hospital with confirmed SARS-CoV-2 infection in UK: national population based cohort study. BMJ. 2020;369:m2107.

6. Emeruwa UN, Ona S, Shaman JL, et al. Associations between built environment, neighborhood socioeconomic status, and SARS-CoV-2 infection among pregnant women in New York City. JAMA. 2020;324:390-392.

7. Emeruwa UN, Spiegelman J, Ona S, et al. Influence of race and ethnicity on severe acute respiratory syndrome coronavirus 2 (SARS-CoV-2) infection rates and clinical outcomes in pregnancy. Obstet Gynecol. 2020;126:1040-1043.

8. Zambrano LD, Ellington S, Strid P, et al; CDC COVID-19 
response pregnancy and infant linked outcomes team. Update: characteristics of symptomatic women of reproductive age with laboratory-confirmed SARS-CoV-2 infection by pregnancy status-United States, January 22-October 3, 2020. MMWR Morb Mortal Wkly Rep. 2020;69:1641-1647.

9. Badr DA, Mattern J, Carlin A, et al. Are clinical outcomes worse for pregnant women at $\geq 20$ weeks' gestation infected with coronavirus disease 2019? A multicenter case control study with propensity score matching. Am J Obstet Gynecol. 2020;223:764-768

10. DeBolt CA, Bianco A, Limaye MA, et al. Pregnant women with severe or critical COVID-19 have increased composite morbidity compared with nonpregnant matched controls. Am J Obstet Gynecol. 2020;S0002-9378:31312-0.

11. Collin J, Byström E, Carnahan A, et al. Public Health Agency of Sweden's Brief Report: pregnant and postpartum women with severe acute respiratory syndrome coronavirus 2 infection in intensive care in Sweden. Acta Obstet Gynecol Scand. 2020;99: 819-822.

12. Dumitriu D, Emeruwa UN, Hanft E, et al. Outcomes of neonates born to mothers with severe acute respiratory syndrome coronavirus 2 infection at a large medical center in New York City. JAMA Pediatr. 2020;e204298.

13. Salvatore CM, Han JY, Acker KP, et al. Neonatal management and outcomes during the COVID-19 pandemic: an observational cohort study. Lancet Child Adolesc Health. 2020;4: 721-727.

14. Shanes ED, Mithal LB, Otero S, et al. Placental pathology in COVID-19. Am J Clin Path. 2020;154:23-32.

15. Centers for Disease Control and Prevention. Duration of isolation and precautions for adults with COVID-19. Updated October 19, 2020. https://www.cdc.gov/corona virus/2019-ncov/hcp/duration-isolation.html?CDC _AA_refVal=https\%3A\%2 F\%2Fwww.cdc.gov\%2F coronavirus\%2F2019-ncov\%2Fcommunity\%2Fstrategy -discontinue-isolation.html. Accessed December 15, 2020.

16. Centers for Disease Control and Prevention.
Discontinuation of transmission-based precautions and disposition of patients with COVID-19 in healthcare settings. Updated August 10, 2020. https://www.cdc.gov /coronavirus/2019-ncov/hcp/disposition-hospitalized -patients.html. Accessed December 15, 2020.

17. Rasmussen SA, Lyerly AD, Jamieson DJ. Delaying pregnancy during a public health crisis-examining public health recommendations for COVID-19 and beyond. $N$ Engl J Med. 2020;383:2097-2099.

18. Reale SC, Field KG, Lumbreras-Marquez MI, et al. Association between number of in-person health care visits and SARS-CoV-2 infection in obstetrical patients. JAMA. 2020;324: 1210-1212.

19. Polack FP, Thomas SJ, Kitchin N, et al. Safety and efficacy of the BNT 162b2 mRNA Covid-19 vaccine. $N$ Engl J Med. December 10, 2020. doi: 10.1056/NEJMoa2034577.

20. Widge AT, Rouphael NG, Jackson LA, et al. Durability of responses after SARS-CoV-2 mRNA-1273 vaccination. December 3, 2020. doi: 10.1056/NEJMc2032195.

21. USFood and Drug Administration. FDAtakes additional action in fight against COVID-19 by issuing emergency use authorization for second COVID-19 vaccine. December 18, 2020 https://www.fda.gov/news-events/press-announcements /fda-takes-additional-action-fight-against-covid-19-issuing -emergency-use-authorization-second-covid. Accessed December 22, 2020

22. American College of Obstetricians and Gynecologists. Prac tice advisory: vaccinating pregnancy and lactating patients against COVID-19. https://www.acog.org/clinical/clinical -guidance/practice-advisory/articles/2020/12/vaccinating -pregnant-and-lactating-patients-against-covid-19. Last updated December 21, 2020. Accessed December 21, 2020.

23. Dooling K, McClung N, Chamberland M, et al. The Advisory Committee on Immunization Practices' interim recom mendation for allocating initial supplies of COVID-19 vaccine-United States, 2020. MMWR Morb Mortal Wkly Rep. 2020;69:1857-1859. 